\title{
TO EVALUATE THE COMPARISON OF INTUBATING CONDITIONS USING FENTANYL PLUS PROPOFOL VERSUS NALBUPHINE PLUS PROPOFOL DURING FIBEROPTIC INTUBATION
}

\author{
Simrit Kaur 1 , Davinder Chawla², Parmod Kumar³, Amandeep Kaur ${ }^{4}$ \\ ${ }_{1}^{1}$ Assistant Professor, Department of Anaesthesia, Government Medical College, Patiala, Punjab, India. \\ ${ }^{2}$ Associate Professor, Department of Anaesthesia, Government Medical College, Patiala, Punjab, India. \\ 3 Professor and HOD, Department of Anaesthesia, Government Medical College, Patiala, Punjab, India. \\ ${ }^{4}$ Senior Resident, Department of Anaesthesia, Government Medical College, Patiala, Punjab, India.
}

\begin{tabular}{l}
\hline ABSTRACT \\
\hline BACKGROUND \\
Awake nasal or oral flexible fiberoptic intubation (AFOI) is the airway management technique of choice in known or anticipated \\
difficult airway, severe cervical stenosis, Chiari malformation, unstable cervical fracture, limited mouth opening as in \\
temporomandibular disease, mandibular-maxillary fixation, severe facial burn and vertebral artery insufficiency.[1] Fiberoptic \\
intubation is the best, easiest and most successful method for awake intubation.
\end{tabular}

\section{MATERIALS AND METHODS}

A prospective, comparative and randomized study was conducted on 100 patients undergoing elective surgery under general anaesthesia. Patients were randomly divided into two groups of 50 each. Group I: Patient received bolus of I.V. fentanyl $1 \mathrm{ug} / \mathrm{kg}+$ propofol $1 \mathrm{mg} / \mathrm{kg}$ so as to achieve an adequate level of sedation i.e. RSS=3. Group II: Patient received bolus of I.V. nalbuphine 0.2 $\mathrm{mg} / \mathrm{kg}+$ propofol $1 \mathrm{mg} / \mathrm{kg}$ to achieve adequate level of sedation. Haemodynamic parameters (heart rate, systolic and diastolic blood pressure, mean arterial pressure), SpO2, EtCO2, total comfort scale and patient's tolerance were assessed during preoxygenation, fiberscope insertion and endotracheal intubation.

\section{RESULTS}

Significant difference $(\mathrm{p}<0.05)$ between two groups in terms of HR, SBP, DBP, MAP, total comfort score and patient tolerance was seen during fiberscope insertion and endotracheal intubation.

\section{CONCLUSION}

Fentanyl plus propofol regimes are suitable for fiberoptic intubation. Fentanyl plus propofol appeared to offer better tolerance, preservation of an airway and spontaneous ventilation, while maintaining haemodynamic stability.

\section{KEY WORDS}

Patient's Tolerance, Ramsay Sedation Score, Haemodynamic Parameters, Total Comfort Scale.

HOW TO CITE THIS ARTICLE: Kaur S, Chawla D, Kumar P, et al. To evaluate the comparison of intubating conditions using fentanyl plus propofol versus nalbuphine plus propofol during fiberoptic intubation. J. Evolution Med. Dent. Sci. 2018;7(51):54305436, DOI: $10.14260 /$ jemds/2018/1202

\section{BACKGROUND}

Awake nasal or oral flexible fiberoptic intubation (AFOI) is the airway management technique of choice in known or anticipated difficult airway, severe cervical stenosis, Chiari malformation, unstable cervical fracture, limited mouth opening as in temporomandibular disease, mandibularmaxillary fixation, severe facial burn and vertebral artery insufficiency.[1] Tracheal intubation is the placement of a flexible plastic tube into the trachea to maintain a patent airway for ventilation. Fiberoptic intubation is the best, easiest and most successful method for awake intubation. The main aim of awake intubation is to have a calm and cooperative patient who can follow verbal commands while maintaining adequate oxygenation and ventilation.

'Financial or Other Competing Interest': None.

Submission 16-03-2018, Peer Review 26-11-2018,

Acceptance 04-12-2018, Published 17-12-2018.

Corresponding Author:

Dr. Davinder Chawla,

Associate Professor,

Department of Anaesthesia,

Government Medical College,

Patiala, Punjab, India.

E-mail: chawladavinder64@yahoo.com

DOI: $10.14260 /$ jemds $/ 2018 / 1202$
Good topical anaesthesia is essential to obtund the sensory afferent stimuli of the oropharyngeal and laryngotracheal region. Establishing topical anaesthesia before intubation prevents coughing, swallowing, laryngospasm and excessive salivation. Both optimal intubating conditions and patient comfort are necessary while preparing the patient for fiberoptic intubation. An ideal sedative is expected to provide comfort and elicit patient cooperation while maintaining haemodynamic stability and spontaneous ventilation. Drugs used for sedation during awake fiberoptic intubation include fentanyl, midazolam, ketamine, propofol, dexmedetomidine, remifentanil etc.[2-5]

Fiberoptic intubation can be performed awake under sedation with or without topical anaesthesia or with muscle relaxant (Suxamethonium, vecuronium, rocuronium, atracurium). For sedation, drugs that may be used are fentanyl/opioids, midazolam, propofol, dexmedetomidine, remifentanil. Each drug has advantages and disadvantages. Fentanyl or other narcotics reduce the discomfort and haemodynamic changes associated with airway instrumentation.[6] In order to compare the different drugs for fiberoptic laryngoscopy which produces better intubating conditions with minimal side effects or no side effects, the present study was conducted to compare the intubating 
condition using fentanyl plus propofol versus nalbuphine plus propofol during fiberoptic intubation.

\section{MATERIALS AND METHODS}

The present prospective, comparative and randomized study was conducted in department of Anaesthesia and intensive care, Government Medical College, Rajindra Hospital, Patiala, after obtaining the approval from ethical committee and after informed consent from all the patients. The study was conducted on 100 patients of either gender aged between 18 to 60 years of age belonging to ASA-I or II and scheduled for elective surgery under general anaesthesia.

\section{Sample Size}

Was estimated based on pilot study; mean difference in heart rate in two groups was 8.75 with SD of 15.06 . With this our sample size $n=47$ per group at a power of $80 \%$ and confidence interval of $95 \%$ with $\mathrm{z}$ value of 1.96 . For possible dropouts, it was decided to include 50 patients per group.

$\mathrm{n}=\left(\mathrm{Z}_{\alpha / 2}+\mathrm{Z}_{\beta}\right)^{2 *} 2^{*} \sigma^{2} / \mathrm{d}^{2}$, where $\mathrm{Z}_{\alpha / 2}$ is the critical value of the normal distribution at $\alpha / 2, Z_{\beta}$ is the critical value of the normal distribution at $\beta, \sigma^{2}$ is the population variance and $d$ is the difference between two means.

All the patients were randomly divided into two groups by simple envelope method. Patients were kept fasting for atleast 6 hours preoperatively. Preanaesthetic check-up was done in every patient including detailed history, clinical examination and necessary investigations. All patients received Inj. Glycopyrrolate $(0.2 \mathrm{mg}) \mathrm{i} / \mathrm{m} 30 \mathrm{~min}$ before the elective surgery. Viscous gargles with $2 \%$ lignocaine was done to achieve adequate topical anaesthesia. Patient shifted to ot. Patient placed in supine position. I.V. line secured. Monitors attached to view $\mathrm{HR}, \mathrm{BP}, \mathrm{SpO}_{2}$ and $\mathrm{EtCO}_{2}$. Nasal mucosa was sprayed with vasoconstrictor xylometazoline $0.1 \%$. Each nostril was checked for patency. The nostril with least resistance was chosen for nasal intubation. Nasal mucosa was sprayed with 2 puffs of $10 \%$ lignocaine. For further topical anaesthesia 2 puffs of $10 \%$ lignocaine was sprayed to tonsillar pillars and back of throat.
Nasopharyngeal dilator with lignocaine jelly was introduced in each nostril. Transtracheal block was given with $3 \mathrm{ml}$ of $4 \%$ lignocaine. The local anaesthetic dose was not exceeding $4 \mathrm{mg} / \mathrm{kg} .{ }^{[7]} \mathrm{A}$ more rapid delivery of local anaesthetic reduces the risk of needle induced trauma due to coughing. [8]

\section{Statistical Analysis}

Analysis was conducted using IBMM SPSS statistics (version 22.0). Statistical Analysis is done by using Chi square test, means of 2 continuous normally distributed variables were compared by independent samples, Student's t-test.

\section{Group I}

Patient received bolus of I.V. fentanyl $1 \mathrm{ug} / \mathrm{kg}+$ propofol 1 $\mathrm{mg} / \mathrm{kg}$ so as to achieve an adequate level of sedation.

\section{Group II}

Patient received bolus of I.V. nalbuphine $0.2 \mathrm{mg} / \mathrm{kg}+$ propofol $1 \mathrm{mg} / \mathrm{kg}$ to achieve adequate level of sedation i:e RSS $=3$.

Lidocaine jelly applied to fiberscope and nostril. Under the guidance of video, fiberscope advanced. On reaching the carina, endotracheal tube was railroaded over the fibrescope. Tracheal placement confirmed by locating murphy's eye during removing the fiberscope. Fiberoptic nasotracheal intubation done in both groups of patients. Duration of fiberoptic intubation was different in different patients ranging from 1 to 5 minutes. Once tracheal intubation was completed and the tube was secured, general anaesthesia was administered. Clinically patients were monitored, and following observation was recorded during the course of intubation-

1. Comfort scale values were recorded by the anaesthesiologist during pre- oxygenation, at point of introduction of fiberscope and at the introduction of ET tube.

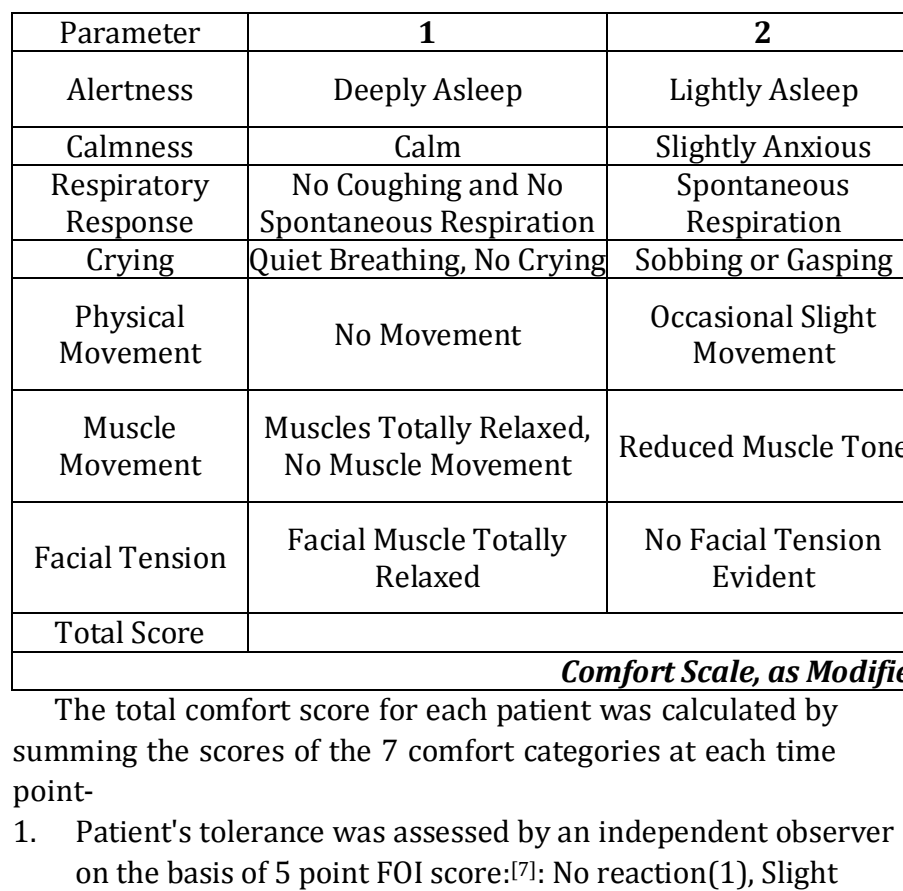




\section{RESULTS}

\section{Demographic Parameters}

The two groups, group I (fentanyl+propofol) and group || (nalbuphine+propofol) were comparable in terms of baseline demographic parameters like age ( $t$ test), Gender (chi square test).

\begin{tabular}{|c|c|c|c|c|c|c|}
\hline \multirow{2}{*}{ Time Interval } & $\begin{array}{c}\text { Group I } \\
\text { (Fentanyl + } \\
\text { Propofol) }\end{array}$ & $\begin{array}{c}\text { Group II } \\
\text { (Nalbuphine + } \\
\text { Propofol) }\end{array}$ & value & Value & Signi \\
ficance & Mean & SD \pm & Mean & SD \pm & & \\
\cline { 2 - 5 } $\begin{array}{c}\text { Baseline Heart } \\
\text { Rate }\end{array}$ & 86.98 & 10.56 & 87.76 & 10.54 & 0.948 & NS \\
\hline $\begin{array}{c}\text { During Pre- } \\
\text { Oxygenation } \\
\text { Heart Rate }\end{array}$ & 86.76 & 9.59 & 88.66 & 10.80 & 0.516 & NS \\
\hline Heart Rate Baseline Heart Rate (Per Min.) and during Pre- \\
Oxygenation
\end{tabular}

There was no significant difference in HR between two groups during baseline and pre-oxygenation.

\begin{tabular}{|c|c|c|c|c|c|c|}
\hline \multirow{2}{*}{\begin{tabular}{|c|} 
Time \\
Interval \\
(per \\
minute)
\end{tabular}} & \multicolumn{2}{|c|}{$\begin{array}{c}\text { Group I } \\
\text { (Fentanyl + } \\
\text { Propofol) } \\
\end{array}$} & \multicolumn{2}{|c|}{$\begin{array}{c}\text { Group II } \\
\text { (Nalbuphine + } \\
\text { Propofol) }\end{array}$} & \multirow[t]{2}{*}{ p-Value } & \multirow[t]{2}{*}{$\begin{array}{l}\text { Signi- } \\
\text { ficance }\end{array}$} \\
\hline & Mean & SD \pm & Mean & SD \pm & & \\
\hline $1 \mathrm{~min}$. & 89.74 & 10.02 & 93.28 & 10.66 & 0.180 & NS \\
\hline $2 \mathrm{~min}$. & 90.82 & 10.34 & 96.32 & 10.57 & 0.038 & $\mathrm{~S}$ \\
\hline $3 \mathrm{~min}$. & 91.42 & 10.63 & 98.52 & 10.54 & 0.004 & $\mathrm{HS}$ \\
\hline $4 \mathrm{~min}$. & 91.88 & 10.43 & 100.06 & 9.80 & $<0.001$ & $\mathrm{HS}$ \\
\hline $5 \mathrm{~min}$. & 92.16 & 10.48 & 101.08 & 9.00 & $<0.001$ & $\mathrm{HS}$ \\
\hline
\end{tabular}

On comparing the heart rate between two groups, no significant differences were found at $1 \mathrm{~min}$. interval $(p>0.05)$ during fiberscope insertion. At 2 min. interval significant difference was found and at 3, 4, 5 mins. interval highly significant difference was found between both the groups.

\begin{tabular}{|c|c|c|c|c|c|c|}
\hline \multirow{2}{*}{\begin{tabular}{|c|} 
Time \\
Interval \\
(per \\
minute)
\end{tabular}} & \multicolumn{2}{|c|}{$\begin{array}{c}\text { Group I } \\
\text { (Fentanyl + } \\
\text { Propofol) }\end{array}$} & \multicolumn{2}{|c|}{$\begin{array}{c}\text { Group II } \\
\text { (Nalbuphine+ } \\
\text { Propofol) }\end{array}$} & \multirow[t]{2}{*}{ p-Value } & \multirow[t]{2}{*}{$\begin{array}{l}\text { Signi- } \\
\text { ficance }\end{array}$} \\
\hline & Mean & SD \pm & Mean & SD \pm & & \\
\hline 1 min. & 92.76 & 10.57 & 103.22 & 8.84 & $<0.001$ & HS \\
\hline $2 \mathrm{~min}$. & 91.66 & 10.26 & 103.70 & 9.50 & $<0.001$ & $\mathrm{HS}$ \\
\hline $3 \mathrm{~min}$. & 89.94 & 9.17 & 104.56 & 9.40 & $<0.001$ & HS \\
\hline $4 \mathrm{~min}$. & 88.74 & 8.81 & 105.08 & 9.07 & $<0.001$ & HS \\
\hline $5 \mathrm{~min}$. & 87.38 & 8.56 & 104.78 & 9.90 & $<0.001$ & $\mathrm{HS}$ \\
\hline \multicolumn{7}{|c|}{ Mean Heart Rate During ET } \\
\hline
\end{tabular}

On comparing the heart rate between two groups highly significant difference were found at 1, 2, 3, 4, 5 mins intervals during endotracheal tube placement. $(\mathrm{p}<0.05)$.

Our study shows no significant difference in mean heart rate between two groups at baseline and preoxygenation but shows significant difference in mean heart rate during FOS and endotracheal tube placement.

Kay et al[10] (1985) conducted study for comparison of fentanyl and nalbuphine for blocking the circulatory responses to tracheal intubation and found significant rise in heart rate in nalbuphine group.

Smith et al in[11] 1992 conducted a study to see effect of fentanyl on the circulatory responses to orotracheal fiberoptic intubation and Macintosh intubation and concluded that fentanyl appears to have useful place in attenuating the cardiovascular effects of fiberoptic intubation under general anaesthesia.

Khan et al[12] (2002) conducted study on comparative evaluation of fentanyl and nalbuphine in total intravenous anaesthesia with propofol infusion in laparoscopic surgery and found that heart rate response was significantly higher in nalbuphine group.

Bhandari et al[13](2014) conducted a comparative study of attenuation haemodynamic responses to intubation using fentanyl and nalbuphine and found that nalbuphine group had increased heart rate during intubation..

Our study concluded that rise in heart rate during fiberoptic intubation was more in nalbuphine and propofol group as compared to fentanyl and propofol group.

\section{Blood Pressure}

Systolic Blood Pressure

\begin{tabular}{|c|c|c|c|c|c|c|}
\hline \multirow{2}{*}{$\begin{array}{c}\text { Time } \\
\text { Interval }\end{array}$} & $\begin{array}{c}\text { Group I } \\
\text { (Fentanyl + } \\
\text { Propofol) }\end{array}$ & $\begin{array}{c}\text { Group II } \\
\text { (Nalbuphine } \\
\text { + Propofol) }\end{array}$ & $\begin{array}{c}\text { p } \\
\text { value }\end{array}$ & $\begin{array}{c}\text { Signi= } \\
\text { ficance }\end{array}$ \\
\cline { 2 - 6 } & Mean & SD \pm & Mean & SD \pm & & \\
\hline Baseline SBP & 128.58 & 10.50 & 124.88 & 10.34 & 0.107 & NS \\
\hline $\begin{array}{c}\text { During Pre- } \\
\text { oxygenation } \\
\text { SBP }\end{array}$ & 127.86 & 8.75 & 127.48 & 10.12 & 0.986 & NS \\
\hline \multicolumn{7}{|c|}{ Mean Baseline SBP and during Pre-Oxygenation } \\
\hline
\end{tabular}

There was no significant difference between the two groups. ( $\mathrm{p}>0.05)$.

\begin{tabular}{|c|c|c|c|c|c|c|}
\hline \multirow{2}{*}{$\begin{array}{c}\text { Time } \\
\text { Interval }\end{array}$} & $\begin{array}{c}\text { Group I } \\
\text { (Fentanyl + } \\
\text { Propofol) }\end{array}$ & $\begin{array}{c}\text { Group II } \\
\text { (Nalbuphine } \\
\text { +Propofol) }\end{array}$ & \multirow{2}{*}{ p-Value } & 倡ni- \\
ficance \\
\cline { 2 - 5 } & Mean & SD \pm & Mean & SD \pm & & \\
\hline 1 min. & 128.94 & 7.96 & 132.62 & 9.61 & 0.031 & S \\
\hline 2 min. & 130.16 & 8.34 & 136.50 & 8.76 & $<0.001$ & HS \\
\hline 3 min. & 130.22 & 7.85 & 140.06 & 7.77 & $<0.001$ & HS \\
\hline 4 min. & 130.88 & 7.57 & 142.12 & 7.94 & $<0.001$ & HS \\
\hline 5 min. & 129.96 & 8.01 & 143.94 & 7.95 & $<0.001$ & HS \\
\hline \multicolumn{7}{|c|}{ Mean SBP during FOS } \\
\hline
\end{tabular}

Significant difference was found in the SBP measurements during Fiberscope insertion between the two groups. $(\mathrm{p}<0.05)$. At $1 \mathrm{~min}$. interval and highly significant difference was found between both groups at 2-, 3-, 4- and 5-min. intervals.

\begin{tabular}{|c|c|c|c|c|c|c|}
\hline \multirow[t]{2}{*}{$\begin{array}{c}\text { Time } \\
\text { Interval }\end{array}$} & \multicolumn{2}{|c|}{$\begin{array}{c}\text { Group I } \\
\text { (Fentanyl + } \\
\text { Propofol) }\end{array}$} & \multicolumn{2}{|c|}{$\begin{array}{c}\text { Group II } \\
\text { (Nalbuphine+ } \\
\text { Propofol) }\end{array}$} & \multirow[t]{2}{*}{ p-Value } & \multirow[t]{2}{*}{$\begin{array}{l}\text { Signi- } \\
\text { ficance }\end{array}$} \\
\hline & Mean & SD \pm & Mean & SD \pm & & \\
\hline $1 \mathrm{~min}$. & 128.78 & 7.28 & 144.96 & 8.57 & $<0.001$ & HS \\
\hline $2 \mathrm{~min}$. & 128.30 & 6.78 & 147.02 & 9.26 & $<0.001$ & HS \\
\hline $3 \mathrm{~min}$. & 128.72 & 7.79 & 147.66 & 8.69 & $<0.001$ & HS \\
\hline $4 \mathrm{~min}$. & 127.60 & 7.54 & 148.12 & 9.81 & $<0.001$ & HS \\
\hline $5 \mathrm{~min}$. & 127.50 & 7.25 & 148.92 & 10.31 & $<0.001$ & $\mathrm{HS}$ \\
\hline \multicolumn{7}{|c|}{ Mean SBP during ET } \\
\hline
\end{tabular}

Highly significant difference was found in the SBP measurements during endotracheal tube placement between the two groups. $(\mathrm{p}<0.05)$. The systolic blood pressure in our study shows no significant difference among two groups during baseline and preoxygenation but shows highly 
significant difference in SBP between two groups during FOS and during ETT placement.

\section{Diastolic Blood Pressure}

\begin{tabular}{|c|c|c|c|c|c|c|}
\hline \multirow{2}{*}{ Time Interval } & \multicolumn{2}{|c|}{$\begin{array}{c}\text { Group I } \\
\text { (Fentanyl + } \\
\text { Propofol) }\end{array}$} & $\begin{array}{c}\text { Group II } \\
\text { (Nalbuphine } \\
\text { +Propofol) }\end{array}$ & $\begin{array}{c}\text { p- } \\
\text { Value }\end{array}$ & $\begin{array}{c}\text { Signi- } \\
\text { ficance }\end{array}$ \\
\cline { 2 - 6 } Mean & SD \pm & Mean & SD \pm & & \\
\hline Baseline DBP & 80.38 & 8.09 & 79.26 & 7.19 & 0.672 & NS \\
\hline $\begin{array}{c}\text { During Pre- } \\
\text { Oxygenation } \\
\text { DBP }\end{array}$ & 80.50 & 7.82 & 81.34 & 6.41 & 0.627 & NS \\
\hline \multicolumn{2}{|l|}{ Mean baseline DBP and during Pre-Oxygenation } \\
\hline
\end{tabular}

There was no significant difference between the two groups. ( $\mathrm{p}>0.05)$.

\begin{tabular}{|c|c|c|c|c|c|c|}
\hline \multirow{2}{*}{$\begin{array}{c}\text { Time } \\
\text { Interval }\end{array}$} & $\begin{array}{c}\text { Group I } \\
\text { (Fentanyl + } \\
\text { Propofol) }\end{array}$ & $\begin{array}{c}\text { Group II } \\
\text { (Nalbuphine } \\
\text { +Propofol) }\end{array}$ & p-Value & Signi- \\
& ficance \\
& Mean & SD \pm & Mean & SD \pm & & \\
\hline 1 min. & 82.18 & 8.05 & 84.54 & 6.81 & 0.094 & NS \\
\hline 2 min. & 81.96 & 8.01 & 87.08 & 6.09 & $<0.001$ & HS \\
\hline 3 min. & 82.90 & 7.82 & 89.72 & 6.39 & $<0.001$ & HS \\
\hline 4 min. & 83.54 & 8.12 & 90.66 & 5.62 & $<0.001$ & HS \\
\hline 5 min. & 82.86 & 8.38 & 90.56 & 5.17 & $<0.001$ & HS \\
\hline \multicolumn{7}{|c|}{ Mean DBP during FOS } \\
\hline
\end{tabular}

At 1 min interval no significant difference was found between both groups, but highly significant difference was found in the DBP measurements during fiberscope insertion $2,3,4,5 \mathrm{~min}$. intervals between the two groups. $(\mathrm{p}<0.05)$.

\begin{tabular}{|c|c|c|c|c|c|c|}
\hline \multirow[t]{2}{*}{$\begin{array}{c}\text { Time } \\
\text { Interval }\end{array}$} & \multicolumn{2}{|c|}{$\begin{array}{c}\text { Group I } \\
\text { (Fentanyl + } \\
\text { Propofol) } \\
\end{array}$} & \multicolumn{2}{|c|}{$\begin{array}{c}\text { Group II } \\
\text { (Nalbuphine + } \\
\text { Propofol) }\end{array}$} & \multirow[t]{2}{*}{$p$ value } & \multirow[t]{2}{*}{$\begin{array}{l}\text { Signi- } \\
\text { ficance }\end{array}$} \\
\hline & Mean & SD \pm & Mean & SD \pm & & \\
\hline $1 \mathrm{~min}$ & 81.52 & 8.63 & 92.98 & 6.02 & $<0.001$ & HS \\
\hline $2 \min$ & 81.58 & 8.21 & 93.48 & 5.93 & $<0.001$ & HS \\
\hline $3 \mathrm{~min}$ & 82.44 & 8.44 & 93.40 & 5.68 & $<0.001$ & HS \\
\hline $4 \mathrm{~min}$ & 81.96 & 7.93 & 93.12 & 5.68 & $<0.001$ & HS \\
\hline $5 \mathrm{~min}$ & 81.62 & 7.43 & 94.24 & 5.16 & $<0.001$ & $\mathrm{HS}$ \\
\hline \multicolumn{7}{|c|}{ Mean DBP during ET } \\
\hline
\end{tabular}

Highly significant difference was found in the DBP measurements during endotracheal tube placement at times $1,2,3,4,5$ min interval $(\mathrm{p}<0.05)$

Our study shows no significant difference in DBP between both groups during baseline and preoxygenation but shows highly significant difference between both the groups during FOS and ETT placement.

\section{Mean Arterial Pressure}

\begin{tabular}{|c|c|c|c|c|c|c|}
\hline \multirow{2}{*}{$\begin{array}{c}\text { Time } \\
\text { Interval }\end{array}$} & \multicolumn{2}{c|}{$\begin{array}{c}\text { Group I } \\
\text { (Fentanyl + } \\
\text { Propofol) }\end{array}$} & $\begin{array}{c}\text { Group II } \\
\text { (Nalbuphine+ } \\
\text { Propofol) }\end{array}$ & $\begin{array}{c}\text { p } \\
\text { value }\end{array}$ & $\begin{array}{c}\text { Signi- } \\
\text { ficance }\end{array}$ \\
\cline { 2 - 6 } & Mean & SD \pm & Mean & SD \pm & & \\
\hline Baseline MAP & 96.16 & 8.77 & 94.24 & 7.27 & 0.145 & NS \\
\hline $\begin{array}{c}\text { During Pre- } \\
\text { oxygenation } \\
\text { MAP }\end{array}$ & 96.20 & 7.32 & 96.34 & 7.26 & 0.844 & NS \\
\hline \multicolumn{7}{|c|}{ Mean Baseline MAP and during Pre-Oxygenation } \\
\hline
\end{tabular}

There was no significant difference between the two groups. ( $\mathrm{p}>0.05)$

\begin{tabular}{|c|c|c|c|c|c|c|}
\hline \multirow{2}{*}{$\begin{array}{c}\text { Time } \\
\text { Interval }\end{array}$} & $\begin{array}{c}\text { Group I } \\
\text { (Fentanyl + } \\
\text { Propofol) }\end{array}$ & $\begin{array}{c}\text { Group II } \\
\text { (Nalbuphine + } \\
\text { Propofol) }\end{array}$ & p-Value & $\begin{array}{c}\text { Signi- } \\
\text { ficance }\end{array}$ \\
\cline { 2 - 6 } & Mean & SD \pm & Mean & SD \pm & & \\
\hline 1 min. & 97.84 & 7.38 & 100.40 & 6.89 & 0.070 & NS \\
\hline 2 min. & 98.20 & 7.00 & 102.98 & 6.13 & 0.001 & HS \\
\hline 3 min. & 98.38 & 7.06 & 106.16 & 5.74 & $<0.001$ & HS \\
\hline 4 min. & 99.56 & 7.23 & 107.62 & 5.31 & $<0.001$ & HS \\
\hline 5 min. & 98.42 & 7.69 & 108.06 & 5.09 & $<0.001$ & HS \\
\hline \multicolumn{7}{|c|}{ Mean MAP during FOS } \\
\hline
\end{tabular}

No significant difference was found in MAP measurements at $1 \mathrm{~min}$. interval between both groups and highly significant difference was found in the MAP measurements during fiberscope insertion 2, 3, 4, $5 \mathrm{~min}$. intervals between the two groups. $(\mathrm{p}<0.05)$.

\begin{tabular}{|c|c|c|c|c|c|c|}
\hline \multirow{2}{*}{$\begin{array}{c}\text { Time } \\
\text { Interval }\end{array}$} & $\begin{array}{c}\text { Group I } \\
\text { (Fentanyl + } \\
\text { Propofol) }\end{array}$ & $\begin{array}{c}\text { Group II } \\
\text { (Nalbuphine + } \\
\text { Propofol) }\end{array}$ & p-Value & $\begin{array}{c}\text { Signi- } \\
\text { ficance }\end{array}$ \\
\cline { 2 - 6 } & Mean & SD \pm & Mean & SD \pm & & \\
\hline 1 min. & 96.86 & 8.37 & 109.78 & 5.86 & $<0.001$ & HS \\
\hline 2 min. & 97.24 & 7.28 & 110.76 & 6.18 & $<0.001$ & HS \\
\hline 3 min. & 97.88 & 7.60 & 111.00 & 5.40 & $<0.001$ & HS \\
\hline 4 min. & 97.30 & 7.16 & 111.20 & 5.85 & $<0.001$ & HS \\
\hline 5 min. & 96.36 & 6.90 & 111.96 & 5.61 & $<0.001$ & HS \\
\hline \multicolumn{7}{|c|}{ Mean MAP during ET } \\
\hline
\end{tabular}

Highly significant difference was found in the MAP measurements during endotracheal tube placement at 1, 2, 3, 4, 5 min. intervals $(\mathrm{p}<0.05)$.

Our findings demonstrate that there was no significant difference in MAP measurements during baseline and preoxygenation, but highly significant difference was found between both the groups during FOS and ETT placement.

Kay et al[10] (1985) conducted study on comparative evaluation of fentanyl and nalbuphine for blocking the circulatory response to tracheal intubation and found significant increase in blood pressure in nalbuphine group as compared to fentanyl group.

Khan et al[12] (2002) had compared nalbuphine and fentanyl as total intravenous anaesthesia with propofol infusion in laparoscopic surgery and found significant increase in systolic blood pressure and diastolic blood pressure in nalbuphine group as compared to fentanyl group.

Freye et al[14] (2001) conducted study to compare cardiovascular response to laryngoscopy and tracheal intubation with fentanyl and nalbuphine and found that significant increase in systolic blood pressure in nalbuphine group as compared to fentanyl.

Sharma et al (2014) conducted a comparative study of haemodynamic response to intubation with fentanyl and nalbuphine and found significant rise in systolic and diastolic blood pressure in nalbuphine group as compared to fentanyl group.

Prashad et al[15](2016) conducted a comparative study of analgesic potential of nalbuphine versus fentanyl during general anaesthesia and found significant increase in systolic blood pressure in nalbuphine group as compared to fentanyl.

Our study concluded that rise in systolic, diastolic and mean blood pressure during fiberoptic intubation was more 
in nalbuphine+propofol group as compared to fentanyl+ propofol group.

\section{Total Comfort Score}

\begin{tabular}{|c|c|c|c|c|c|c|}
\hline \multirow{2}{*}{$\begin{array}{c}\text { Time } \\
\text { Interval }\end{array}$} & $\begin{array}{c}\text { Group I } \\
\text { (Fentanyl + } \\
\text { Propofol) }\end{array}$ & $\begin{array}{c}\text { Group II } \\
\text { (Nalbuphine+ } \\
\text { Propofol) }\end{array}$ & p-Value & Signifi \\
cance & Mean & SD \pm & Mean & SD \pm & & \\
\cline { 2 - 6 } $\begin{array}{c}\text { During Pre } \\
\text { Oxygenation }\end{array}$ & 12.86 & 1.22 & 12.66 & 1.18 & 0.746 & NS \\
\hline During FOS & 14.00 & 1.90 & 14.96 & 1.32 & 0.001 & S \\
\hline During ET & 15.20 & 2.08 & 16.06 & 1.05 & 0.022 & S \\
\hline \multicolumn{7}{|c|}{ Total Comfort Score (TCS) } \\
\hline
\end{tabular}

The Total Comfort Score when calculated showed a significant difference between the two groups during FOS and ET. Our study shows that fentanyl and propofol provided better intubating conditions as compared to nalbuphine and propofol

Rai et al[16] (2008) in their study remifentanil targetcontrolled infusion vs propofol target controlled infusion for conscious sedation for awake fibreoptic intubation observed better intubating conditions with remifentanil as compared to propofol.

Dhasmana et al[17] ( 2010) also observed in their study awake blind nasotracheal intubation in temporomandibular joint ankylosis patients under conscious sedation using fentanyl and midazolam that these drugs provide better intubating conditions in awake nasotracheal intubation.

Rodrigues et al[18] (2013) conducted study on difficult airway intubation with flexible bronchoscope on 102 patients and concluded that fiberoptic bronchoscope intubation in conscious sedation protocol with midazolam and fentanyl provides better intubating conditions and was safe in the management of patients with difficult airway.

Our study also concludes that fentanyl and propofol provides better intubating conditions as compared to nalbuphine and propofol.

\section{Patients' Tolerance to the Placement of Tube}

\begin{tabular}{|c|c|c|c|c|c|c|}
\hline \multirow{2}{*}{$\begin{array}{c}\text { Time } \\
\text { Interval }\end{array}$} & $\begin{array}{c}\text { Group I } \\
\text { (Fentanyl + } \\
\text { Propofol) }\end{array}$ & $\begin{array}{c}\text { Group II } \\
\text { (Nalbuphine + } \\
\text { Propofol) }\end{array}$ & $\begin{array}{c}\text { p- } \\
\text { Value }\end{array}$ & $\begin{array}{c}\text { Signi- } \\
\text { ficance }\end{array}$ \\
\cline { 2 - 5 } & Mean & SD \pm & Mean & SD \pm & & \\
\hline FOS & 3.28 & 0.72 & 3.56 & 0.61 & 0.035 & S \\
\hline ET & 2.38 & 0.53 & 2.64 & 0.56 & 0.028 & S \\
\hline \multicolumn{7}{|c|}{ Patient's Tolerance based on 5 Point Fiberoptic Index } \\
Score \\
\hline
\end{tabular}

Significant difference between two groups $(\mathrm{p}<0.05)$ was found suggesting that fentanyl and propofol had better patient tolerability.

\section{DISCUSSION}

Our study shows no significant difference in mean heart rate between two groups at baseline and preoxygenation but shows significant difference in mean heart rate during FOS and endotracheal tube placement.

Kay et al[10] (1985) conducted study for comparison of fentanyl and nalbuphine for blocking the circulatory responses to tracheal intubation and found significant rise in heart rate in nalbuphine group.
Smith et al in[11] 1992 conducted a study to see effect of fentanyl on the circulatory responses to orotracheal fiberoptic intubation and Macintosh intubation and concluded that fentanyl appears to have useful place in attenuating the cardiovascular effects of fiberoptic intubation under general anaesthesia.

Khan et al[12] (2002) conducted study on comparative evaluation of fentanyl and nalbuphine in total intravenous anaesthesia with propofol infusion in laparoscopic surgery and found that heart rate response was significantly higher in nalbuphine group.

Bhandari et al[13](2014) conducted a comparative study of attenuation haemodynamic responses to intubation using fentanyl and nalbuphine and found that nalbuphine group had increased heart rate during intubation..

Our study concluded that rise in heart rate during fiberoptic intubation was more in nalbuphine and propofol group as compared to fentanyl and propofol group

Our findings demonstrate that there was no significant difference in MAP measurements during baseline and preoxygenation, but highly significant difference was found between both the groups during FOS and ETT placement.

Kay et al[10] (1985) conducted study on comparative evaluation of fentanyl and nalbuphine for blocking the circulatory response to tracheal intubation and found significant increase in blood pressure in nalbuphine group as compared to fentanyl group.

Khan et al[12] (2002) had compared nalbuphine and fentanyl as total intravenous anaesthesia with propofol infusion in laparoscopic surgery and found significant increase in systolic blood pressure and diastolic blood pressure in nalbuphine group as compared to fentanyl group.

Freye et al[14] (2001) conducted study to compare cardiovascular response to laryngoscopy and tracheal intubation with fentanyl and nalbuphine and found that significant increase in systolic blood pressure in nalbuphine group as compared to fentanyl.

Sharma et al[89](2014) conducted a comparative study of haemodynamic response to intubation with fentanyl and nalbuphine and found significant rise in systolic and diastolic blood pressure in nalbuphine group as compared to fentanyl group.

Prashad et al[15](2016) conducted a comparative study of analgesic potential of nalbuphine versus fentanyl during general anaesthesia and found significant increase in systolic blood pressure in nalbuphine group as compared to fentanyl.

Our study concluded that rise in systolic, diastolic and mean blood pressure during fiberoptic intubation was more in nalbuphine+propofol group as compared to fentanyl+propofol group

Our study shows that fentanyl and propofol provided better intubating conditions as compared to nalbuphine and propofol

Rai et al[16] (2008) in their study remifentanil targetcontrolled infusion vs propofol target controlled infusion for conscious sedation for awake fibreoptic intubation observed better intubating conditions with remifentanil as compared to propofol.

Dhasmana et al[17] (2010) also observed in their study awake blind nasotracheal intubation in temporomandibular joint ankylosis patients under conscious sedation using 
fentanyl and midazolam that these drugs provide better intubating conditions in awake nasotracheal intubation.

Rodrigues et al[18] (2013) conducted study on difficult airway intubation with flexible bronchoscope on 102 patients and concluded that fiberoptic bronchoscope intubation in conscious sedation protocol with midazolam and fentanyl provides better intubating conditions and was safe in the management of patients with difficult airway.

Significant difference between two groups $(\mathrm{p}<0.05)$ was found suggesting that fentanyl and propofol had better patient tolerability.

Dhasmana et al[17] (2010) also observed in their study awake blind nasotracheal intubation in temporomandibular joint ankylosis patients under conscious sedation using fentanyl and midazolam that these drugs provide better patient comfort and sedation in awake nasotracheal intubation.

Rodrigues et al[18] (2013) conducted study on difficult airway intubation with flexible bronchoscope on 102 patients and concluded that fiberoptic bronchoscope intubation according to the conscious sedation protocol with midazolam and fentanyl effective and safe in the management of patients with difficult airway.

\section{CONCLUSION}

Fiberoptic intubation remains the accepted standard in elective airway management of the awake spontaneously breathing patient with an anticipated difficult airway.[23] Fiberoptic intubation proves beneficial as it avoids mechanical stimulus to oropharynx and larynx and attenuates pressor response to tracheal intubation. Fiberoptic nasotracheal intubation was done in both groups of patients. Mean Heart Rate measured during fiberoptic intubation showed statistically significant increase in heart rate with nalbuphine $(0.2 \mathrm{mg} / \mathrm{kg})$ and propofol $(1 \mathrm{mg} / \mathrm{kg})$ than fentanyl $(1 \mu \mathrm{g} / \mathrm{kg})$ and propofol (1 mg/kg). SBP, DBP and MAP measured during fiberoptic intubation showed statistically significant increase with nalbuphine $(0.2 \mathrm{mg} / \mathrm{kg})$ and propofol $(1 \mathrm{mg} / \mathrm{kg})$ than fentanyl $(1 \mu \mathrm{g} / \mathrm{kg})$ and propofol $(1 \mathrm{mg} / \mathrm{kg})$. $\mathrm{SpO}_{2}$ values during preoxygenation, fiberscope insertion and endotracheal intubation was not significant in both groups. No episode of apnoea was noted in any of the patients. Patients in fentanyl and propofol group (Group I) felt more comfortable during AFOI than nalbuphine and propofol group (Group II). No significant difference was found in $\mathrm{EtCO}_{2}$ between both groups. Patients who received fentanyl and propofol (Group I) had better tolerance to AFOI than those who received nalbuphine and propofol (Group II).

To conclude, the use of fentanyl $(1 \mu \mathrm{g} / \mathrm{kg})$ and propofol $(1$ $\mathrm{mg} / \mathrm{kg}$ ) is safe and effective in patients undergoing fiberoptic intubation offering conscious sedation, better tolerance and comfort while maintaining oxygen saturation without any haemodynamic alteration while on the other side use of nalbuphine $(0.2 \mathrm{mg} / \mathrm{kg})$ and propofol $(1 \mathrm{mg} / \mathrm{kg})$ during fiberoptic intubation causes significant increase in heart rate, blood pressure and provides less tolerance and comfort.

\section{REFERENCES}

[1] Artime C. Flexible fiberoptic intubation. In: Hagberg CA, Daily WH, eds. The difficult airway: a practical guide. Oxford University Press 2013.

[2] Lallo A, Billard V, Bourgain JL. A comparison of propofol and remifentanil target-controlled infusions to facilitate fiberoptic nasotracheal intubation. Anaesthesia and Analgesia 2009;108(3):852-7.

[3] Stamenkovic DM, Hassid M. Dexmedetomidine for fiberoptic intubation of a patient with severe mental retardation and atlantoaxial instability. Acta Anaethesiologica Scandinavica 2006;50(10):1314-5.

[4] Knolle E, Oehmke MJ, Gustorff B, et al. Targetcontrolled infusion of propofol for fibreoptic intubation. European Journal of Anaesthesiology 2003;20(7):565-9.

[5] Machata AM, Gonano C, Holzer A, et al. Awake nasotracheal fibreoptic intubation: patient comfort, intubating condition and haemodynamic stability during conscious sedation with remifentanil. Anesth Analg 2003;97(3):904-8.

[6] WCPI Focus on Pain Series. The three faces of fentanyl. http://aspi.wisc.edu/wpi/focus/sping96.htm.

[7] Sutherland AD, Williams RT. Cardiovascular responses and lidocaine absorption in fiberoptic - assisted awake intubation. Anesth Analg 1986;65(4):389-91.

[8] Furlan JC. Anatomical study applied to anesthetic block technique of the superior laryngeal nerve. Acta Anaesthesiol Scand 2002;46(2):199-202.

[9] Ambuel B, Hamlett KW, Marx CM, et al. Assessing distress in pediatric intensive care environments: the COMFORT scale. J Pediatr Psychol 1992;17(1):95-109.

[10] Kay B, Healy TE, Bolder PM. Blocking the circulatory responses to tracheal intubation. A comparison of fentanyl and nalbuphine. Anaesthesia 1985;40(10):960-3.

[11] Smith JE, King MJ, Yanny HF, et al. Effect of fentanyl on the circulatory responses to orotracheal fibreoptic intubation. Anaesthesia 1992;47(1):20-3.

[12] Khan FA, Hameedullah. Comparison of fentanyl and nalbuphine in total intravenous anaesthesia (TIVA). J Pak Med Assoc 2002;52(10):459-65.

[13] Sharma N, Parikh H. A comparative study of haemodynamic responses to intubation: fentanyl versus nalbuphine. Gujarat Medical Journal 2014;69(2):48-53.

[14] Freye E, Levy JV. Reflex activity caused by laryngoscopy and intubation is obtunded differently by meptazinol, nalbuphine and fentanyl. Eur J Anaesthesiol 2007;24(1):53-8.

[15] Prasad HK, Kumar RPA, Rajagokilam R, et al. A comparative study of analgesic potential of nalbuphine versus fentanyl during general anaesthesia. International Journal of Contemporary Medical Research 2016;3(10):2815-8.

[16] Rai MR, Parry TM, Dombrovskis A, et al. Remifentanil target-controlled infusion vs propofol targetcontrolled infusion for conscious sedation for awake fibreoptic intubation: a double-blinded randomized controlled trial. Bri J Anaesth 2008;100(1):125-30. 
[17] Dhasmana S, Singh V, Pal US. Awake blind nasotracheal intubation in temporomandibular joint ankylosis patients under conscious sedation using fentanyl and midazolam. Journal of Maxillofacial \& Oral Surgery 2010;9(4):377-81.
[18] Rodrigues AJ, Scordamagilo PR, Palomino AM, et al. Difficult airway intubation with flexible bronchoscope. Bras J Anesthesiol 2013;63(4):358-61. 